\title{
THE 14-DIMENSIONAL KERVAIRE INVARIANT AND THE SPORADIC GROUP $\mathrm{M}_{12}$ *
}

\author{
R. JAMES MILGRAM ${ }^{\dagger}$
}

There is a 14 dimensional aspect to the sporadic group $M_{12}$. For one thing

$$
\operatorname{Syl}_{2}\left(M_{12}\right)=\operatorname{Syl}_{2}\left(G_{2}(q)\right)
$$

for $q \equiv 3,5 \bmod (8)$ and the exceptional Lie group $G_{2}$ is 14 dimensional. Secondly, in [AMM1] we showed that the Poincaré series for $H^{*}\left(M_{12} ; \mathbb{F}_{2}\right)$ is

$$
\frac{1+t^{2}+3 t^{3}+t^{4}+3 t^{5}+4 t^{6}+2 t^{7}+4 t^{8}+3 t^{9}+t^{10}+3 t^{11}+t^{12}+t^{14}}{\left(1-t^{4}\right)\left(1-t^{6}\right)\left(1-t^{7}\right)}
$$

which suggests, since $\frac{1}{\left(1-t^{4}\right)\left(1-t^{6}\right)\left(1-t^{7}\right)}$ is the Poincaré series for the classifying space $B_{G_{2}}$, that there should be a fibering of the form

$$
F \longrightarrow B_{M_{12}}^{+} \longrightarrow B_{G_{2}}
$$

with the fiber a 14 dimensional Poincaré duality space. Indeed, in [M], (see also [BW]), I constructed just such a fibration.

There is one more way in which one might expect the sporadic nature of $M_{12}$ to manifest itself. According to [R], the natural homotopy class in

$$
\pi_{14}^{s}\left(S^{0}\right)=2^{2}=\left\langle\bar{\kappa}, \sigma^{2}\right\rangle
$$

associated to the reframing of $G_{2}$ is $\bar{\kappa}$, so one would expect that there was some way in which a homotopy class in $\pi_{14}^{s}\left(S^{0}\right)$ could be associated to $F$, and this class should be $\sigma^{2}$ or $\sigma^{2}+\bar{\kappa}$. In other words, this class should represent an element in $\pi_{14}^{s}\left(S^{0}\right)$ of Kervaire invariant one. Indeed, it is the object of this note to show just that.

One connection between homotopy theory and group theory lies in the well known equivalence $\mathbb{Z} \times B_{\mathcal{S}_{\infty}}^{+} \simeq Q\left(S^{0}\right)=\lim _{n \mapsto \infty}\left(\Omega^{n} \Sigma^{n}\right)$, and there is a natural embedding $h: M_{12} \subset \mathcal{S}_{12}$, embedding $M_{12}$ as a maximal subgroup of $\mathcal{S}_{12}$, that gives rise to a composition map

$$
B_{h}: B_{M_{12}}^{+} \longrightarrow B_{\mathcal{S}_{12}}^{+} \hookrightarrow B_{\mathcal{S}_{\infty}}^{+} \simeq Q\left(S^{0}\right)
$$

which, at the homotopy level induces a map $B_{h, *}: \pi_{*}\left(B_{M_{12}}^{+}\right) \rightarrow \pi_{*}^{s}\left(S^{0}\right)$. Then the main result of this note is

Theorem. There is an element $A \in \pi_{14}^{s}\left(B_{M_{12}}\right)$ with Hurewicz image $[F]$, the orientation class of the fiber $F$ above, and every such $A$ has image $B_{h}(A)=\sigma_{7}^{2}$ or $\sigma_{7}^{2}+\bar{\kappa}$ under the composition above.

The way in which the class $A$ is constructed may have independent interest. The construction is based on the use of the stable transfer,

$$
\operatorname{tr}: Q\left(B_{G_{2}(q)}\right) \longrightarrow Q\left(B_{S y l_{2}\left(M_{12}\right)}\right)
$$

*Received May 17, 1997; accepted for publication September 15, 1997. Partially supported by a grant from the N.S.F..

†Department of Mathematics, Stanford University, Stanford, CA, 94305, U.S.A. (milgram@ gauss.stanford.edu). 
the existence of a fibration

$$
G_{2} \longrightarrow B_{G_{2}(q)}^{+} \longrightarrow B_{G_{2}}
$$

and the fact that $G_{2}$ is parallelizable to construct the homotopy class $A$, while the inclusions $S_{y l}\left(M_{12}\right) \hookrightarrow M_{12} \hookrightarrow \mathcal{S}_{12}$ give rise to an exotic way of mapping the homotopy class associated to $G_{2}$ into stable homotopy.

There is yet one more connection we should mention. In [MO] we construct stable splittings for $B_{M_{12}}$ and $B_{S y l_{2}\left(M_{12}\right)}$. In particular, $B_{M_{12}}$ splits into irreducible summands as follows:

$$
B_{M_{12}} \simeq_{s} B_{G_{2}(3)} \vee B_{L_{3}(2)} \vee X_{E, S_{1}}
$$

where $E=Q_{8} * Q_{8} \subset S y l_{2}\left(M_{12}\right)$ and $X_{E, S_{1}}$ is one of the three dominant summands of this group. Then, by looking at the proof of our main result it becomes clear that the result would not have been possible without the summand $B_{L_{3}(2)}$ being part of the decomposition. In particular this tends to indicate that if there are any further direct constructions of Kervaire invariant one classes, the group $G$ involved must, at the very least, have non-trivial Schur multiplier since $H_{2}\left(B_{L_{3}(2)} ; \mathbb{F}_{2}\right) \neq 0$.

All homology and cohomology groups in the remainder of this note will have coefficients $\mathbb{F}_{2}$ and so the coefficients will not appear in the expressions $H^{*}(X)$ or $H_{*}(X)$.

In $\S 1$ we review the basic facts we need about $M_{12}, G_{2}(q)$, and $S y l_{2}\left(M_{12}\right)$, as well as the cohomology of these groups. In particular, Theorem 1.8 gives the structure of $H^{*}(F)$ and proves the important relation $\left\langle\alpha^{7},[F]\right\rangle=1$ where $\alpha$ is the two dimensional generator in $H^{*}\left(M_{12}\right)$.

THEOREM 1.8. $H^{*}(F)$ has generators $\alpha, \beta, c, m$, and $n$, subject to the following relations: $m^{4}=n^{2}=0, \alpha^{2} \beta^{2}=\left(\alpha^{3}+\beta^{2}\right) \beta=0, \alpha^{5}=\alpha^{2} \beta c, \alpha^{7}=m^{3} n$. The Steenrod operations are given on these generators by $S q^{1}(\alpha)=\beta, S q^{1}(c)=\alpha^{2}, S q^{2}(\beta)=\alpha \beta$, $S q^{2}(c)=\alpha(\beta+c), S q^{1}(m)=0, S q^{2}(m)=n, S q^{4}(n)=0$. A table of the generators is given as follows

$\begin{array}{cccccccccccccc}\operatorname{Dim} & 2 & 3 & 4 & 5 & 6 & 7 & 8 & 9 & 10 & 11 & 12 & 13 & 14 \\ & \alpha & \beta & \alpha^{2} & \alpha \beta & \alpha^{3} & & \alpha^{4} & & & & & & \\ & c & & \alpha c & \beta c & \alpha^{2} c & \alpha \beta c & \alpha^{3} c & & \alpha^{4} c & & & \\ & & & & \beta^{2} & \alpha^{2} \beta & \alpha \beta^{2} & \alpha^{3} \beta & \alpha^{5} & \alpha^{4} \beta & \alpha^{6} & & \\ & m & & n & m^{2} & & m n & m^{3} & & m^{2} n & & & \alpha^{7}=m^{3} n\end{array}$

and these generators also form a basis for $H^{*}\left(M_{12}\right)$ as a module over the Dickson algebra $D_{3}=\mathbb{F}_{2}\left[d_{4}, d_{6}, d_{7}\right]$.

It is exactly the fact that $\alpha^{7}$ is dual to $[F]$ which twists the homotopy class $A$ to a Kervaire invariant one class. The relation between $G_{2}$ and $F$ reminds one of a degree one surgery problem, but I don't know if $F$ can be replaced by a closed manifold.

In $\S 2$ and $\S 3$ we review the basic facts we need from iterated loop space theory. Then, in $\S 4$ we determine the cohomology map $H^{*}\left(S_{12}\right) \rightarrow H^{*}\left(M_{12}\right)$ induced from the inclusion above. This also may have independent interest, though the idea is very direct. Since both groups are detected by restriction to maximal elementaries it suffices to analyze the conjugacy classes of the images of the three maximal $2^{3}$ 's in $M_{12}$. The result is given in table (4.5) and the explicit images of the generators are then given exactly in the remarks following the table.

Finally, in $\S 5$ we put all this together to prove our main result. Here, the key step is an explicit determination of a transfer map $\operatorname{tr}^{*}\left(H^{*}\left(Q\left(B_{M_{12}}\right)\right) \rightarrow H^{*}\left(Q\left(B_{G_{2}(q)}\right)\right)\right.$ as far 
as we need it. Again this is achieved by restricting to 2-elementaries. The key result, Lemma 5.3, which gives an explicit method for determining such transfer-restriction may also have independent interest.

1. The groups $M_{12}$ and $G_{2}(q)$ and their common 2-Sylow subgroup. The cohomology of $M_{12}$ is discussed in [AM, pp. 261-263]. It is detected by restriction to the three $2^{3}$ 's which represent the three conjugacy classes of maximal elementary 2-groups in $M_{12}$. The result is

THEOREM 1.1. $H^{*}\left(M_{12} ; \mathbb{F}_{2}\right)$ has 8 generators, $\alpha_{2}, \beta_{3}, c_{3}, m_{3}, d_{4}, n_{5}, d_{6}$ and $d_{7}$, (where the subscripts denote the dimension) with restriction images given as follows:

\begin{tabular}{|c|c|c|c|}
\hline gen. \group & $V_{1}$ & $V_{3}$ & $V_{5}$ \\
\hline$\alpha$ & 0 & $h^{2}$ & $d_{2}$ \\
\hline$\beta$ & 0 & 0 & $d_{3}$ \\
\hline$c$ & 0 & $h^{3}$ & $\tau^{3}+\tau^{2} \lambda+\lambda^{3}$ \\
\hline$m$ & $d_{3}$ & 0 & 0 \\
\hline$d_{4}$ & $d_{4}$ & $d_{4}$ & $d_{4}$ \\
\hline$n$ & $d_{2} d_{3}$ & 0 & 0 \\
\hline$d_{6}$ & $d_{6}$ & $d_{6}$ & $d_{6}$ \\
\hline$d_{7}$ & $d_{7}$ & $d_{7}$ & $d_{7}$ \\
\hline
\end{tabular}

where

$$
\begin{aligned}
H^{*}\left(2^{3}\right) & =\mathbb{F}_{2}[h, \tau, \lambda], \\
d_{2} & =\tau^{2}+\tau \lambda+\lambda^{2}, \\
d_{3} & =\tau^{2} \lambda+\lambda^{2} \tau \\
d_{4} & =h^{4}+h^{2} d_{2}+h d_{3}+d_{2}^{2}, \\
d_{6} & =h^{4} d_{2}+h^{2} d_{2}^{2}+h d_{2} d_{3}+d_{3}^{2}, \\
d_{7} & =h^{4} d_{3}+h^{2} d_{2} d_{3}+h d_{3}^{2} .
\end{aligned}
$$

The structure of $H^{*}\left(B_{G_{2}(q)}\right)$ with $q \equiv 3,5 \bmod (8)$ is described in [M] similarly. The groups share the same 2-Sylow subgroup but $G_{2}(q)$ has exactly two maximal 2elementaries, the first represented by $V_{3}$ while $V_{1}$ fuses with $V_{5}$ to give the second. In particular, $H^{*}\left(G_{2}(q) ; \mathbb{F}_{2}\right)$ is also represented by restriction to $V_{1}$ and $V_{3}$, and we have the result of $[\mathrm{M}]$

Theorem 1.4. $H^{*}\left(B_{G_{2}(q)}\right)$ with $q \equiv 3,5 \bmod (8)$ has 5 generators $\bar{m}_{3}, d_{4}, \bar{n}_{5}, d_{6}$, $d_{7}$, with $\bar{m}_{3}$ restricting to $d_{3}$ in $H^{*}\left(V_{1}\right), H^{*}\left(V_{5}\right)$, and 0 in $H^{*}\left(V_{3}\right), \bar{n}=S q^{2}(\bar{m})$, and the $d_{i}$ as above.

There is an identification of $\lim _{n \mapsto \infty} B_{G_{2}\left(q^{n}\right)}^{+}$localized at 2 with $B_{G_{2}}$ localized at 2 via etale theory and consequently a (2-local) fibration

$$
G_{2} \longrightarrow B_{G_{2}(q)}^{+} \longrightarrow B_{G_{2}}
$$

using a theorem of J. Harper which identifies $G_{2}$ (2-locally) with any simply connected space which has the same $\mathbb{F}_{2}$-cohomology ring as $G_{2}$ as a module over the Steenrod algebra. Moreover we obtain the cohomology of the fiber of the map $B_{G_{2}(q)}^{+} \rightarrow B_{G_{2}}$ by 
noting that since $H^{*}\left(B_{G_{2}(q)} ; \mathbb{F}_{2}\right)$ is Cohen-Macaulay over $H^{*}\left(B_{G_{2}} ; \mathbb{F}_{2}\right)=\mathbb{F}_{2}\left[d_{4}, d_{6}, d_{7}\right]$, it follows that

$$
H^{*}(\text { Fiber })=H^{*}\left(B_{G_{2}(q)}\right) \otimes_{\mathbb{F}_{2}\left[d_{4}, d_{6}, d_{7}\right]} \mathbb{F}_{2}
$$

and this is seen to give the desired isomorphism (see e.g. the discussion below, specifically the remarks preceeding 1.8).

Relating the two restriction images we see that $\bar{m} \mapsto m+\beta$ in $H^{*}\left(M_{12}\right), \bar{n} \mapsto n+$ $\alpha \beta$ and $H^{*}\left(G_{2}(q)\right) \subset H^{*}\left(M_{12}\right)$ though there is no homomorphism $M_{12} \rightarrow G_{2}(q)$ which will induce this cohomology map. However, in [M] a 2-local map of +-constructions

$$
B_{M_{12}}^{+} \stackrel{f}{\longrightarrow} B_{G_{2}(q)}^{+}
$$

was constructed which does, in fact, realize the cohomology map above. In particular we get a diagram of fiberings

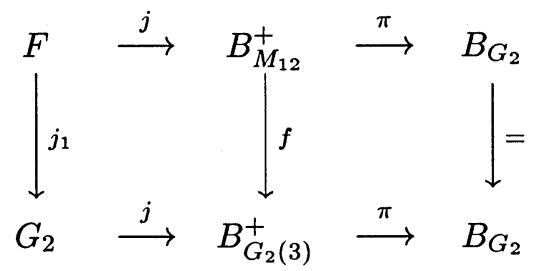

Again, in the upper fibration, since $H^{*}\left(B_{M_{12}}^{+} ; \mathbb{F}_{2}\right)$ is Cohen-Macaulay over $H^{*}\left(B_{G_{2}}\right)$ from $[\mathrm{AM}]$, we have

$$
H^{*}(F)=H^{*}\left(B_{M_{12}}\right) \otimes_{\mathbb{F}_{2}\left[d_{4}, d_{6}, d_{7}\right]} \mathbb{F}_{2}
$$
$\bar{m}^{2}$.

It is well known that $H^{*}\left(G_{2}\right)=\mathbb{F}_{2}\left[\bar{m}_{3}\right] /\left(\bar{m}^{4}\right) \otimes E(\bar{n})$ with $S q^{2}(\bar{m})=\bar{n}, S q^{1}(\bar{n})=$

We also need the structure of $H^{*}(F)$ as a module over the Steenrod algebra. The result is

TheOREM 1.8. $H^{*}(F)$ has generators $\alpha, \beta, c, m$, and $n$, subject to the following relations: $m^{4}=n^{2}=0, \alpha^{2} \beta^{2}=\left(\alpha^{3}+\beta^{2}\right) \beta=0, \alpha^{5}=\alpha^{2} \beta c, \alpha^{7}=m^{3} n$. The Steenrod operations are given on these generators by $S q^{1}(\alpha)=\beta, S q^{1}(c)=\alpha^{2}, S q^{2}(\beta)=\alpha \beta$, $S q^{2}(c)=\alpha(\beta+c), S q^{1}(m)=0, S q^{2}(m)=n, S q^{4}(n)=0$. A table of the generators is given as follows

$$
\begin{aligned}
& \begin{array}{llllllllllllll}
\operatorname{Dim} & 2 & 3 & 4 & 5 & 6 & 7 & 8 & 9 & 10 & 11 & 12 & 13 &
\end{array} \\
& \begin{array}{cccccccccc}
\alpha & \beta & \alpha^{2} & \alpha \beta & \alpha^{3} & & \alpha^{4} & & \\
& c & & \alpha c & \beta c & \alpha^{2} c & \alpha \beta c & \alpha^{3} c & \alpha^{4} c
\end{array} \\
& \begin{array}{llllllll}
\beta^{2} & \alpha^{2} \beta & \alpha \beta^{2} & \alpha^{3} \beta & \alpha^{5} & \alpha^{4} \beta & \alpha^{6}
\end{array} \\
& m \quad n \quad m^{2} \quad m n \quad m^{3} \quad m^{2} n \quad \alpha^{7}=m^{3} n
\end{aligned}
$$

and these generators also form a basis for $H^{*}\left(M_{12}\right)$ as a module over the Dickson algebra $D_{3}=\mathbb{F}_{2}\left[d_{4}, d_{6}, d_{7}\right]$.

This result does not appear in the published literature, though it is given in [O]. We defer the proof to $\S 6$ since the details of the proof are not needed in proving our main result. 
REMARK 1.9. The element in $H^{*}\left(G_{2}(q)\right)$ which restricts to $\left(0, d_{7}^{2}, 0\right)$ is particularly important in what follows. We have the following decompositions in terms of the generators $\alpha, \beta, m, n, d_{4}, d_{6}$ and $d_{7}$ :

$$
\begin{aligned}
& \left(d_{7}^{2}, 0,0\right)=m^{3} n+m n d_{6}+m d_{4} d_{7} \\
& \left(0,0, d_{7}\right)=\beta d_{4}+\alpha^{2} \beta \\
& \left(0, d_{7}^{2}, 0\right)=d_{7}^{2}+(m+\beta) d_{4} d_{7}+\alpha^{2} \beta d_{7}+m n d_{6}+m^{3} n .
\end{aligned}
$$

We also have a second description of this element as

$$
\left(0, d_{7}^{2}, 0\right)=\alpha\left(d_{6}+\alpha d_{4}+\alpha^{3}+\beta^{2}\right)^{2}
$$

where the first description gives the expression for this class in $H^{*}(F)$ as $m^{3} n$ while the second expression gives it as $\alpha^{7}+\alpha \beta^{4}$. But since, in $H^{*}(F)$ we have $\beta^{3}=\alpha^{3} \beta$ it follows that $\beta^{4}=\alpha(\alpha \beta)^{2}=0$ which establishes the cup product relation $\alpha^{7}=m^{3} n$ in the theorem. Also, this shows that $\alpha^{7}$ evaluates as 1 on the top dimensional class of the fiber $F$ and hence also the fiber $G_{2}$.

For later use we also need to recall some of the detailed structure of $S_{y l} l_{2}\left(M_{12}\right)$, which from here on we denote $H$.

$$
H \cong 4^{2}: 2^{2}=\langle c, d\rangle:\langle s, k\rangle
$$

where the action is given by $c^{s}=d, d^{s}=c, c^{k}=c^{-1}, d^{k}=d^{-1}$. Then $H$ has five conjugacy classes of maximal 2-elementary subgroups

$$
\begin{aligned}
& V_{1}=\left\langle c^{2}, d^{2}, k\right\rangle \\
& V_{2}=\left\langle c^{2}, d^{2}, c k\right\rangle \\
& V_{3}=\left\langle c^{2}, d^{2}, c d k\right\rangle \\
& V_{4}=\left\langle(c d)^{2}, k, s\right\rangle \\
& V_{5}=\left\langle(c d)^{2}, c d k, s\right\rangle
\end{aligned}
$$

with $V_{1}$ and $V_{3}$ normal in $H$. Moreover, as already noted, they fuse as follows: in $M_{12},\left\{V_{1}, V_{2}\right\},\left\{V_{3}, V_{4}\right\},\left\{V_{5}\right\}$, and in $G_{2}(q),\left\{V_{1}, V_{2}, V_{5}\right\},\left\{V_{2}, V_{3}\right\}$.

2. Filtration arguments. We briefly review the homology structure of $Q\left(S^{0}\right)$ as far as we need it.

First, $\lim _{n \mapsto \infty} B_{\mathcal{S}_{n}}=B_{\mathcal{S}_{\infty}}$, and there is a map $e: B_{\mathcal{S}_{\infty}} \rightarrow Q\left(S^{0}\right)_{0}$, the 0-component of $Q\left(S^{0}\right)$, which is a homology equivalence. Moreover, the inclusion $B_{\mathcal{S}_{n}} \hookrightarrow B_{\mathcal{S}_{\infty}}$ induces an inclusion in homology.

The loop sum operation

$$
*: Q\left(S^{0}\right) \times Q\left(S^{0}\right) \longrightarrow Q\left(S^{0}\right)
$$

makes $H_{*}\left(Q\left(S^{0}\right)\right)$ into a graded commutative ring, while the inclusions

$$
{ }_{{ }_{m, n}}: \mathcal{S}_{m} \times \mathcal{S}_{n} \hookrightarrow \mathcal{S}_{n+m}
$$

induce maps of classifying spaces

$$
B_{+}: B_{\mathcal{S}_{m}} \times B_{\mathcal{S}_{n}} \hookrightarrow B_{\mathcal{S}_{n+m}}
$$


which make $H_{*}\left(B_{\mathcal{S}_{\infty}}\right)$ into a commutative ring. They fit together in a commutative diagram

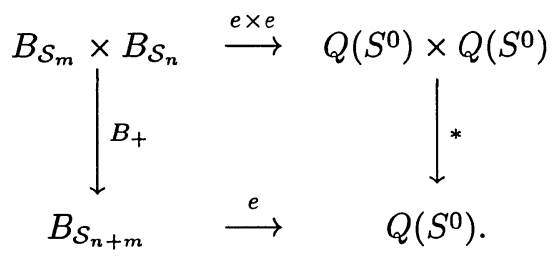

We can put in more structure. There are inclusions $p: \mathcal{S}_{n} \imath 2 \rightarrow \mathcal{S}_{2 n}$ which, at the level of classifying spaces lead to maps

$$
\left(B_{\mathcal{S}_{n}}\right)^{2} \times_{\mathbb{Z} / 2} E_{\mathbb{Z} / 2} \stackrel{B_{p}}{\longrightarrow} B_{\mathcal{S}_{2 n}}
$$

and hence, for each class $\alpha \in H_{j}\left(\mathcal{S}_{n}\right)$, there is an associated class in $H_{2 j+m}\left(B_{\mathcal{S}_{n} 22}\right)$ which, at the chain level is $\alpha \otimes \alpha \otimes e_{m}$. The image of this class in $H_{2 j+m}\left(B_{\mathcal{S}_{2 n}}\right)$ is called $Q_{m}(\alpha)$ and we can iterate this operation obtaining $Q_{\left(i_{1}, \ldots, i_{r}\right)}(\alpha)$ in dimension $2^{r} j+2^{r-1} i_{r}+\cdots+i_{1}$.

REMARK. The construction above actually has an intrinsic description. Let $C_{k}\left(\mathbb{R}^{n}\right)$ be the configuration space of $k$-tuples of ordered, distinct points of $\mathbb{R}^{n}$. Let $f_{1}, \ldots, f_{k}$ be based maps $S^{n}=\mathbb{R}^{n} \cup \infty \stackrel{f_{i}}{\rightarrow} X$, then define, for $\left(z, f_{1}, \ldots, f_{k}\right) \in C_{k}\left(\mathbb{R}^{n}\right) \times$ $\left(\Omega^{n} X\right)^{k}$, the map $g: \mathbb{R}^{n} \cup \infty \rightarrow X$ as $f_{i}$ (suitably rescaled) in a disk neighborhood of $x_{i}$, the $i^{\text {th }}$ point in $z$ and $\infty$ in the complement of these neighborhoods. Now pass to limits over $n$ and note that the construction actually factors through $C_{k}\left(\mathbb{R}^{k}\right) \times \mathcal{S}_{k}\left(\Omega^{n} X\right)^{k}$.

The operation $\alpha \mapsto Q_{i}(\alpha)$ is linear and there is a Cartan formula which connects it with the loop sum:

$$
Q_{i}(\alpha * \beta)=\sum_{j=0}^{i} Q_{j}(\alpha) * Q_{i-j}(\beta)
$$

Let $I=\left(i_{1}, \ldots, i_{r}\right)$. We say that $I$ is admissible if and only if $0 \leq i_{j} \leq i_{j+1}$ for each $j=1, \ldots, r-1$. Note that $Q_{0}(\alpha)=\alpha * \alpha$ the loop sum product. For this reason we suppress those $I$ which contain 0 's and say $I$ is generating if it idmissible and $i_{1}>0$.

Theorem 2.5. $H_{*}\left(B_{\mathcal{S}_{\infty}}\right)=\mathbb{F}_{2}\left[1, Q_{1}(J) * 2(-J), \ldots, Q_{I}(J) * 2^{r}(-J), \ldots\right]$ where the ring structure is generated by loop sum, $J$ represents the limit $J=\lim _{n \rightarrow \infty}\left(J_{n}: S^{n} \rightarrow\right.$ $\left.S^{n}\right)$ with $J_{n}$ the identity map, and $I=\left(i_{1}, \ldots, i_{r}\right)$ runs over all generating sequences.

A filtration degree in $H_{*}\left(B_{\mathcal{S}_{\infty}}\right)$ is given by setting $\operatorname{deg}(a * b)=\operatorname{deg}(a)+\operatorname{deg}(b)$, while $\operatorname{deg}\left(Q_{I}(J)\right)=2^{r}$ for $I=\left(j_{1}, \ldots, j_{r}\right)$ and $\operatorname{deg}(a+b)=\max (\operatorname{deg}(a), \operatorname{deg}(b))$. Then $\mathcal{F}_{n}$ is the subgroup generated by all the elmements of degree $\leq n$ in $H_{*}\left(B_{\mathcal{S}_{\infty}}\right)$. It turns out that $H_{*}\left(B_{\mathcal{S}_{n}}\right)$ is exactly the subgroup consisting of the elements above of filtration $\leq n$.

Dually, let $\mathcal{F}^{n}$ be the kernel of the cohomology surjection $H^{*}\left(B_{\mathcal{S}_{\infty}}\right) \rightarrow H^{*}\left(B_{\mathcal{S}_{n}}\right)$. Then $\mathcal{F}^{n}$ and $\mathcal{F}_{n}$ are mutual annhilators, and $\mathcal{F}^{n}$ is closed under the action of the Steenrod algebra $\mathcal{A}(2)$.

Also, there is a natural compliment to $\mathcal{F}_{n}$, the ideal in $H_{*}\left(B_{\mathcal{S}_{\infty}}\right)$ generated by the elements above with second degree $>n$. In view of the linearity of the $Q_{i}$-operations and the Cartan formula this ideal is actually intrinsic, and independent of the perhaps different choices for embedding $B_{\mathcal{S}_{n}}$ to construct $Q\left(S^{0}\right)$. We call this ideal $\mathcal{H}_{n}$, and this gives an associated graded algebra, $\amalg \mathcal{H}_{n} / \mathcal{H}_{n+1}$. 
Additionally, the composite

$$
\mathcal{F}_{n} \hookrightarrow H_{*}\left(B_{\mathcal{S}_{\infty}}\right) \stackrel{p}{\longrightarrow} H_{*}\left(B_{\mathcal{S}_{\infty}}\right) / \mathcal{H}_{n}
$$

is an isomorphism.

Finally, note that $\mathcal{H}_{n}$ and $\mathcal{F}^{n}$ are dual to each other.

REMARK 2.6. The associated graded ring $\coprod_{j} \mathcal{H}_{j} / \mathcal{H}_{j+1}$ is naturally isomorphic to $H_{*}\left(B_{\mathcal{S}_{\infty}}\right)$ as an algebra but not as a coalgebra. Indeed, as a coalgebra the associated graded ring is primitively generated.

3. The construction of $Q(X)$. We recall the well known May-Milgram construction of $Q(X)=\lim _{n \mapsto \infty} \Omega^{n} \Sigma^{n} X$ for $X$ any connected $C W$-complex with base point $*$.

$$
Q(X) \simeq \coprod_{1}^{\infty} E_{\mathcal{S}_{n}} \times \mathcal{S}_{n} X^{n} / \sim
$$

where $\sim$ is an equivalence relation that identifies points of the form $\left\{e, x_{1}, \ldots, x_{n}\right\}$ with $\left\{p_{i}(e), x_{1}, \ldots, \widehat{x_{i}}, \ldots, x_{n}\right\}$ if $x_{i}=*$, where $p_{i}: E_{\mathcal{S}_{n}} \rightarrow E_{\mathcal{S}_{n-1}}$ is a suitable map. A good model for $E_{\mathcal{S}_{n}}$ is the configuration space $C_{n}\left(\mathbb{R}^{\infty}\right)$ which consists of all ordered $n$-tuples $\left(x_{1}, \ldots, x_{n}\right) \in\left(\mathbb{R}^{\infty}\right)^{n}$ where the $x_{i}$ are all distinct. Of course $\mathcal{S}_{n}$ acts by permuting coordinates. With this model $p_{i}$ simply forgets $x_{i}$.

The natural map $Q(Q(X)) \rightarrow Q(X)$ given as $\Omega^{\infty}($ eval $)$ where

$$
\text { eval: } \Sigma^{n} \Omega^{n} X \rightarrow X
$$

is the usual evaluation map is realized in this model by the inclusion

$$
E_{\mathcal{S}_{n}} \times \mathcal{S}_{n}\left(E_{\mathcal{S}_{m}} \times \mathcal{S}_{m} X^{m}\right)^{n}=E_{\mathcal{S}_{n}} \times\left(E_{\mathcal{S}_{n}}\right)^{n} \times \mathcal{S}_{m} \imath \mathcal{S}_{n} X^{n m} \hookrightarrow E_{\mathcal{S}_{n m}} \times \mathcal{S}_{n m} X^{n m}
$$

obtained by regarding the product of the $E$ 's as a free $\mathcal{S}_{m} \imath \mathcal{S}_{n}$ space and including the wreath product as as subgroup of $\mathcal{S}_{n m}$.

Consequently, given a subgroup $G \subset \mathcal{S}_{n}$ there is a map $B_{G} \stackrel{e}{\hookrightarrow} B_{\mathcal{S}_{n}} \hookrightarrow Q\left(S^{0}\right)$, which prolongs to a map $Q(e): Q\left(B_{G}\right) \rightarrow Q\left(S^{0}\right)$.

The homology of $Q(X)$ is given as

$$
\mathbb{F}_{2}\left[\alpha, \cdots Q_{I}(\alpha), \cdots\right]
$$

as $I$ runs over all generating sequences as above and the $\alpha$ run over a basis for $H_{*}\left(X ; \mathbb{F}_{2}\right)$. Moreover the homology map $H_{*}\left(Q\left(B_{G}\right)\right) \rightarrow H_{*}\left(Q\left(S^{0}\right)\right)$ is determined by observing that it is natural with respect to loop sums and $Q_{I}(\alpha) \mapsto Q_{I}(e(\alpha))$.

As a basic example, suppose that $H \subset G$ has index $k<\infty$. Then there is the Frobenius homomorphism

$$
f: G \longrightarrow H \imath \mathcal{S}_{k}
$$

defined for example in [AM, pp. 73-75] which gives rise to $B_{f}: B_{G} \rightarrow E_{\mathcal{S}_{k}} \times \mathcal{S}_{k}\left(B_{H}\right)^{k}$, and by prolongation, a map

$$
Q\left(B_{G}\right) \stackrel{Q\left(B_{f}\right)}{\longrightarrow} Q\left(B_{H}\right)
$$


which is one of the variants and generalizations of the well known cohomology transfer map.

In particular $H^{*}(H)$ embeds into $H^{*}\left(Q\left(B_{H}\right)\right)$ via the liftings

$$
\alpha \mapsto \alpha \otimes 1 \otimes \cdots \otimes 1+1 \otimes \alpha \otimes \cdots \otimes 1+\cdots+1 \otimes \cdots \otimes 1 \otimes \alpha
$$

taking $H^{*}(H)$ to $H^{*}\left(E_{\mathcal{S}_{k}} \times \mathcal{S}_{k}\left(B_{H}\right)^{k}\right)$, and the composite $B_{G} \hookrightarrow Q\left(B_{G}\right) \stackrel{\text { tr }}{\longrightarrow} Q\left(B_{H}\right)$ gives rise to the ordinary cohomology transfer $H^{*}(H) \stackrel{t r}{\rightarrow} H^{*}(G)$.

4. The inclusion $M_{12} \hookrightarrow \mathcal{S}_{12}$. It is well known, see e.g. [AM, p. 185], that $H^{*}\left(\mathcal{S}_{n}, \mathbb{F}_{p}\right)$ is detected by restriction to conjugacy class representatives of the maximal elementary abelian $p$-groups, and since the same is true for $H^{*}\left(M_{12}\right)$ it suffices to study the induced maps on elementary 2-groups in order to determine the cohomology map $H^{*}\left(\mathcal{S}_{12} ; \mathbb{F}_{2}\right) \rightarrow H^{*}\left(M_{12} ; \mathbb{F}_{2}\right)$.

The conjugacy classes of maximal elementary 2-groups in $\mathcal{S}_{n}$ are also completely determined in [AM, pp. 185-186]. For $\mathcal{S}_{12}$ they are $\left(W_{1}\right)^{6}, W_{2} \times\left(W_{1}\right)^{4},\left(W_{2}\right)^{2} \times\left(W_{1}\right)^{2}$, $\left(W_{3}\right) \times\left(W_{1}\right)^{2},\left(W_{2}\right)^{3}$ and $W_{2} \times W_{3}$ where $W_{i} \cong 2^{i} \subset \mathcal{S}_{2^{i}}$ is the regular representation of $2^{i}$.

The embedding of $M_{12}$ in $\mathcal{S}_{12}$ is given explicitly in [AM, pp. 254-255]. There, if we set $c=d b^{-1}$, we find that a copy of $S y l_{2}\left(M_{12}\right)$ is given as $\langle c, d, k, s\rangle$ and the map into $\mathcal{S}_{12}$ restricts to this subgroup as

$$
\begin{aligned}
c & \mapsto(1,10,11,12)(2,9,3,5) \\
d & \mapsto(1,10,11,12)(4,8,7,6) \\
k & \mapsto(1,12)(5,9)(6,8)(10,11) \\
s & \mapsto(1,11)(2,7)(3,4)(5,8)(6,9)(10,12) .
\end{aligned}
$$

Thus the image of $V_{1}$ is given as

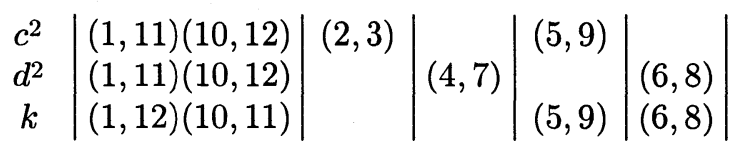

which shows that $V_{1} \hookrightarrow W_{2} \times\left(W_{1}\right)^{4} \subset \mathcal{S}_{12}$. For the second, $V_{3}$, we have

$$
\begin{array}{c|c|c|c|}
c^{2} & 1,11)(10,12) & (2,3)(5,9) & \\
d^{2} & (1,11)(10,12) & & (4,7)(6,8) \\
c d k & (1,10)(11,12) & (2,5)(3,9) & (4,6)(7,8)
\end{array}
$$

which shows that $V_{3} \hookrightarrow\left(W_{2}\right)^{3} \subset \mathcal{S}_{12}$. Finally, the last group $V_{5}$ gives

$$
\begin{array}{c|c|c|}
c^{2} d^{2} & & (2,3)(4,7)(5,9)(6,8) \\
c^{-1} d k & (1,12)(10,11) & (2,9)(3,5)(4,6)(7,8) \\
k s & (1,10)(11,12) & (2,7)(3,4)(5,6)(8,9)
\end{array}
$$

so $V_{5} \hookrightarrow W_{2} \times W_{3} \subset \mathcal{S}_{12}$.

In [AMM2], see also [AM, pp. 213-214], the cohomology ring $H^{*}\left(\mathcal{S}_{12} ; \mathbb{F}_{2}\right)$ was determined, and it was shown that over the Steenrod algebra $\mathcal{A}(2)$ it has five generators 
$\sigma_{1}, \sigma_{2}, \sigma_{3}, \sigma_{4}$, and $\sigma_{6}$. Under restriction we get the following table

\begin{tabular}{|c|c|c|c|}
\hline gen. \group & $W_{2} \times\left(W_{1}\right)^{4}$ & $\left(W_{2}\right)^{3}$ & $W_{2} \times W_{3}$ \\
\hline$\sigma_{1}$ & $1 \otimes \sigma_{1}$ & 0 & 0 \\
\hline$\sigma_{2}$ & $d_{2} \otimes 1+1 \otimes \sigma_{2}$ & $S\left(d_{2} \otimes 1 \otimes 1\right)$ & $d_{2} \otimes 1$ \\
\hline$\sigma_{3}$ & $d_{2} \otimes \sigma_{1}+1 \otimes \sigma_{3}$ & 0 & 0 \\
\hline$\sigma_{4}$ & $d_{2} \otimes \sigma_{2}+1 \otimes \sigma_{4}$ & $S\left(d_{2} \otimes d_{2} \otimes 1\right)$ & $1 \otimes d_{4}$ \\
\hline$\sigma_{6}$ & $d_{2} \otimes \sigma_{4}$ & $d_{2} \otimes d_{2} \otimes d_{2}$ & $d_{2} \otimes d_{4}$ \\
\hline
\end{tabular}

where $\sigma_{i}$ denotes the $i^{\text {th }}$ symmetric monomial in the generators for the individual summands, while $d_{i}$ denotes the appropriate Dickson element in the cohomology of $W_{i}$.

In terms of the filtration discussed in $\S 3 \sigma_{1}^{i}$ is dual to the element $Q_{i}(J) *(-2)$, the unique element in dimension $i$ and filtration 2 but not 4 , while $\sigma_{2}^{i} \sigma_{1}^{k}$ lies in filtration 4 but not 6 and is dual to $e_{i} * e_{i+k}$. Also, $S q^{1}\left(\sigma_{2}\right)+\sigma_{2} \sigma_{1}$ is dual to $Q_{(1,1,)}(J)$ and has filtration 4 but not 6 as do the elements $\left(\sigma_{2}\right)^{i}\left(S q^{1}\left(\sigma^{2}\right)^{j}\right.$ which corresponds to $Q_{j, i+j}(J)$. All the remaining elements of the image have filtration degree at least 6 .

The data above together with these restrictions of the $\sigma_{i}$ generators now determines the restriction image explicitly. As an example we do the cases of $\sigma_{3}$ and $\sigma_{6}$ restricting to $H^{*}\left(V_{1}\right)$. Let $x, y$ generate $H^{*}\left(W_{2}\right), z_{1}, z_{2}, z_{3}, z_{4}$ generate $H^{*}\left(\left(W_{1}\right)^{4}\right)$, in the order above, then we see that

$$
\begin{aligned}
c^{2} & \mapsto x^{*} z_{1}^{*} z_{3}^{*} \\
d^{2} & \mapsto x^{*} z_{2}^{*} z_{4}^{*} \\
k & \mapsto y^{*} z_{3}^{*} z_{4}^{*}
\end{aligned}
$$

so, dually,

$$
\begin{aligned}
x & \mapsto \lambda+\tau \\
y & \mapsto h \\
z_{1} & \mapsto \lambda \\
z_{2} & \mapsto \tau \\
z_{3} & \mapsto \lambda+h \\
z_{4} & \mapsto \tau+h
\end{aligned}
$$

where $h$ is dual to $k, \lambda$ is dual to $c^{2}$, and $\tau$ is dual to $d^{2}$. It follows that we have

$$
\begin{aligned}
d_{2} \otimes \sigma_{1}+1 \otimes \sigma_{3} & \mapsto\left(\lambda \tau(\lambda+\tau)+(\tau+\lambda)\left(\lambda \tau+(\lambda+\tau) h+h^{2}\right)\right. \\
& =(\lambda+\tau)^{2} h+(\lambda+\tau) h^{2} \\
& =d_{3} \\
d_{2} \otimes \sigma_{4} & \mapsto\left((\lambda+\tau)^{2}+h(\lambda+\tau)+h^{2}\right) \lambda \tau(\lambda+h)(\tau+h) \\
& =\lambda \tau\left(h^{4}+\lambda \tau h^{2}+(\lambda+\tau)\left(\lambda^{2}+\tau^{2}+\lambda \tau\right) h+\lambda \tau\left(\lambda^{2}+\tau^{2}\right)\right) \\
& =d_{6}+d_{3}^{2} .
\end{aligned}
$$

The calculations in the remaining cases are similar. Specifically, $\sigma_{1}$ restricts to zero 
in all three, and for the rest we have

\begin{tabular}{|c|c|c|c|}
\hline gen. \group & $V_{1}$ & $V_{3}$ & $V_{5}$ \\
\hline$\sigma_{1}$ & 0 & 0 & 0 \\
\hline$\sigma_{2}$ & 0 & $h^{2}$ & $d_{2}$ \\
\hline$\sigma_{3}$ & $d_{3}$ & 0 & 0 \\
\hline$\sigma_{4}$ & $d_{4}$ & $d_{4}$ & $d_{4}$ \\
\hline$\sigma_{6}$ & $d_{6}+d_{3}^{2}$ & $h^{6}+d_{6}$ & $d_{6}+d_{2}^{3}+d_{3}^{2}$ \\
\hline
\end{tabular}

Hence $\alpha=\operatorname{res}^{*}\left(\sigma_{2}\right), m=\operatorname{res}^{*}\left(\sigma_{3}\right), d_{4}=\operatorname{res}^{*}\left(\sigma_{4}\right), \operatorname{res}^{*}\left(\sigma_{6}\right)=\sigma^{3}+\beta^{2}+m^{2}+d_{6}$. Moreover, since $\beta=S q^{1}(\alpha), n=S q^{2}(m), d_{6}=S q^{2}\left(d_{4}\right)$ and $d_{7}=S q^{1}\left(d_{6}\right)$ it follows that among the generators for $H^{*}\left(M_{12}\right)$ only $c$ is not in the image of restriction.

5. The connection of $M_{12}$ with the Kervaire class in dimension 14. Recall that $H \subset M_{12}$ is the 2-Sylow subgroup of $M_{12}$. The sequence of inclusions

$$
H \hookrightarrow M_{12} \stackrel{h}{\rightarrow} \mathcal{S}_{12}
$$

gives rise to the sequence of inclusions of classifying spaces

$$
B_{H} \hookrightarrow B_{M_{12}} \stackrel{B_{h}}{\longrightarrow} B_{\mathcal{S}_{12}} \hookrightarrow Q\left(S^{0}\right)
$$

which prolongs to

$$
Q\left(B_{H}\right) \hookrightarrow Q\left(B_{M_{12}}\right) \stackrel{Q\left(B_{h}\right)}{\longrightarrow} Q\left(S^{0}\right)
$$

in the manner explained in $\S 3$. On the other hand, as we pointed out in $\S 1, H$ is also the 2-Sylow subgroup of $G_{2}(q)$ for $q \equiv 3,5 \bmod (8)$. Thus we have the transfer map $Q\left(B_{G_{2}(q)}\right) \rightarrow Q\left(B_{H}\right)$, and, since $Q\left(B_{G_{2}(q)}\right)=Q\left(B_{G_{2}(q)}^{+}\right)$because the Whitehead theorem already shows that $\Sigma B_{G} \simeq \Sigma B_{G}^{+}$, we obtain the following sequence of maps:

$$
Q\left(G_{2}\right) \longrightarrow Q\left(B_{G_{2}(q)}^{+}\right) \stackrel{t r}{\longrightarrow} Q\left(B_{H}\right) \longrightarrow Q\left(B_{M_{12}}\right) \longrightarrow Q\left(S^{0}\right) .
$$

But $G_{2}$, being a Lie group, is parallelizable, so the suspension $\Sigma^{14} G_{2} \simeq S^{28} \vee \Sigma^{14} Y$, with $Y$ the 11-skeleton of $G_{2}$, and there is a corresponding spherical class of the form $\left[G_{2}\right]+D \in H_{14}\left(Q\left(G_{2}\right) ; \mathbb{Z}\right)$ where $D$ is a sum of loop sum decomposable elements. Consequently we finally obtain the composition

$$
S^{14} \longrightarrow Q\left(G_{2}\right) \longrightarrow Q\left(B_{G_{2}(q)}^{+}\right) \stackrel{t r}{\longrightarrow} Q\left(B_{H}\right) \longrightarrow Q\left(B_{M_{12}}\right) \longrightarrow Q\left(S^{0}\right)
$$

and we want to determine the image of this $S^{14}$ in $\pi_{14}\left(Q\left(S^{0}\right)\right)=\pi_{14}^{s}\left(S^{0}\right)$.

What we need to understand to begin is the map $t r^{*}: H^{*}(H) \rightarrow H^{*}\left(G_{2}(q)\right)$, at least when restricted to the image of $H^{*}\left(M_{12}\right)$. In $G_{2}(q)$ we have pointed out that for the 5 conjugacy classes of $2^{3}$ 's in $H, V_{1}, V_{2}$ and $V_{5}$ fuse to $V_{I I}$ with normalizer quotient $N_{G_{2}(3)}\left(V_{I I}\right) /\left(V_{I I}\right)=\mathcal{S}_{4}$ (fixing a point) while $V_{3}$ and $V_{4}$ fuse to $V_{I}$ with normalizer quotient $L_{3}(2)$. (In particular, the centralizers of $V_{I I}$ and $V_{I}$ are just the $V_{j}, j=I, I I$ themselves in $G_{2}(3)$.) 
Lemma 5.3. The image of a general element of $H^{*}(H)$ under transfer to the cohomology ring $H^{*}\left(G_{2}(q)\right)$ and then restriction to $H^{*}\left(V_{I I}\right)$ is the sum

$$
c_{g_{1}}^{*} \sum_{\tau_{1}} \tau_{1}^{*}\left(r e s_{H}^{V_{1}}(a)\right)+c_{g_{2}}^{*} \sum_{\tau_{2}} \tau_{2}^{*}\left(\operatorname{res}_{H}^{V_{2}}(a)\right)+\sum_{\tau_{5}} \tau_{5}^{*}\left(\operatorname{res}_{H}^{V_{5}}(a)\right)
$$

where $\tau_{i}$ runs over coset representatives for $N_{G}\left(V_{i}\right) / N_{H}\left(V_{i}\right)$ while $g_{1} \in G_{2}(3)$ satisfies $g_{1}^{-1} V_{5} g_{1}=V_{1}$, and $g_{2}^{-1} V_{5} g_{2}=V_{2}$. Similarly, for transfer through $G_{2}(a)$ followed by restriction to $V_{I}$ we get the following sum

$$
c_{g_{3}} \sum_{\tau_{3}} \tau_{3}^{*}\left(r e s_{H}^{V_{3}}(a)\right)+\sum_{\tau_{4}} \tau_{4}^{*}\left(\operatorname{res}_{H}^{V_{4}}(a)\right)
$$

with $g_{3}^{-1} V_{4} g_{3}=V_{3}$, and $\tau_{i}$ as above.

Proof. We do the case of $V_{5}$, the case of $V_{4}$ being identical. Let $G_{2}(q)=\coprod V_{5} g_{i} H$ be the double coset decomposition. From the Mackey formula we have that the composition $r e s^{*} \cdot t r$ above is the sum over the $g_{i}$ of the compositions

$$
H^{*}(H) \stackrel{\text { res }}{\longrightarrow} H^{*}\left(H \cap g_{i}^{-1} V_{5} g_{i}\right) \stackrel{c_{g}^{*}}{\longrightarrow} H^{*}\left(g_{i}^{-1} H g_{i} \cap V_{5}\right) \stackrel{t r}{\longrightarrow} H^{*}\left(V_{5}\right)
$$

but the last transfer is identically zero unless $g_{i}^{-1} V_{5} g_{i} \subset H$, that is to say, $g_{i}^{-1} V_{5} g_{i}$ is equal to one of $V_{5}, V_{1}$ or $V_{2}$ (this last since we can vary $g_{i}$ by right multiplication by any element in $H$ without changing the double coset).

On the other hand, if $g_{i}^{-1} V_{5} g_{i} \subset H$ then $V_{5} g_{i} H=g_{i}\left(g_{i}^{-1} V_{5} g_{i}\right) H=g_{i} H$ is just an ordinary coset of $H$, and conversely, if $V_{5} g H=g^{\prime} H$ then $g^{-1} V_{5} g \subset H$ so the set of terms in the Mackey formula which are non-zero correspond exactly to the set of ordinary cosets of $H$ in the double coset decompostion of $G_{2}(q)$. We now identify these double cosets more precisely.

Suppose that $g_{i}^{-1} V_{5} g_{i}=g_{j}^{-1} V_{5} g_{j} \subset H$. Then

$$
g_{j}^{-1} g_{i}: g_{i}^{-1} V_{5} g_{i} \longrightarrow g_{i}^{-1} V_{5} g_{i}
$$

is an automorphism of this subgroup of $H$. Varying $g_{i}$ on the left by elements of $V_{5}$ has no effect on the automorphism, while varying $g_{i}$ on the right by elements of $N_{H}\left(g_{i}^{-1} V_{5} g_{i}\right)$ varies the automorphism by an element in $N_{G}\left(g_{i}^{-1} V_{5} g_{i}\right)$ which lies in the same coset of $H$ as $g_{j}^{-1} g_{i}$ in $G_{2}(q)$. Consequently, the set of cosets $g_{i} H$ corresponding to $V_{i}$ is in one to one correspondence with $N_{G}\left(V_{i}\right) / N_{H}\left(V_{i}\right)$, and the result follows.

REMARK 5.6. Actually the lemma above is a special case of (the evident) general formula for the effect of the composition of transfer with restriction to an elementary abelian $p$-group.

Corollary 5.7. The composite $H^{*}(H) \stackrel{t r^{*}}{\rightarrow} H^{*}\left(G_{2}(3)\right) \rightarrow H^{*}\left(V_{I I}\right)$ on an element a which restricts to zero in $H^{*}\left(V_{1}\right)$ and $H^{*}\left(V_{2}\right)$ is the same as the composition

$$
H^{*}(H) \stackrel{r^{r e s}}{\longrightarrow} H^{*}\left(V_{5}\right) \stackrel{\Sigma}{\longrightarrow} H^{*}\left(V_{5}\right)
$$

where $\Sigma\left(\operatorname{res}^{*}(a)\right)=\sum_{t \in \mathcal{S}_{3}} t^{*}\left(\operatorname{res}^{*}(a)\right)$ and $\mathcal{S}_{3}=\mathcal{S}_{4} / N_{H}\left(V_{5}\right)$.

EXAMPLE 5.8. Note that $c \in H^{*}\left(M_{12}\right)$ restricts to an element in $H^{*}\left(V_{5}\right)$ which is invariant under $\mathbb{Z} / 3$ and restricts to 0 in $V_{1}, V_{2}$. Thus, $\operatorname{tr}_{H}^{G_{2}(q)}(c)=\left(1+\tau^{*}\right) c$ where $\tau^{*}$ exchanges $\lambda$ and $\tau$. But this is just $d_{3}$. Hence, $t r^{*}(c)=m$. 
EXAMPLE 5.9. A second special case of (5.4) is the transfer for $c \alpha^{2}$ in dimension 7. In $V_{I I}$ the restriction of $\operatorname{tr}\left(c \alpha^{2}\right)$ is $d_{2}^{2} d_{3}=d_{7}+d_{3} d_{4}$ while in $V_{I}$ the restriction is the sum over the cosets of $D_{8} \subset L_{3}(2)$ of $\tau\left(h^{7}\right)$. This can be evaluated as follows: the cosets are the set of flags $W_{1} \subset W_{2} \subset V_{I}$ where $W_{i}$ is a vector subspace of $V_{I}$ having dimension $i$. In particular we can regard $h$ as representing the non-zero vector in the dual $V_{I}^{*}$ which annihilates $W_{2}$, so, since there are exactly three flags with the same $W_{2}$ we see that

$$
\begin{aligned}
\operatorname{res}_{G_{2}(3)}^{V_{I}} t r^{*}\left(c \alpha^{2}\right) & =3 \sum_{v \in V_{I}} v^{7} \\
& =d_{7}
\end{aligned}
$$

so it follows that $t r^{*}\left(c \alpha^{2}\right)=m d_{4}+d_{7} \in H^{*}\left(G_{2}(3)\right)$. Similarly, we have

$$
\operatorname{tr}^{*}\left(\alpha^{7}\right)=m^{3} n \bmod \left(I\left(\mathbb{F}_{2}\left[d_{4}, d_{6}, d_{7}\right]\right)\right)
$$

where $I\left(\mathbb{F}_{2}\left[d_{4}, d_{6}, d_{7}\right]\right)$ is the augmentation ideal in the Dickson algebra, since it restricts to 0 in $H^{*}\left(V_{I I}\right)$ and $d_{7}^{2} \in H^{*}\left(V_{I}\right)$.

Finally, we can determine the image of transfer on each of the remaining generators of $H^{*}\left(M_{12}\right) \subset H$ in $H^{*}\left(G_{2}(3)\right)$ as a module over the Dickson algebra. This is all we need to determine the cohomology map trores*: $H^{*}\left(M_{12}\right) \rightarrow H^{*}\left(G_{2}(q)\right)$ since

$$
\operatorname{tr}_{H}^{G}\left(a \cup r e s_{G}^{H}(b)\right)=\operatorname{tr}_{H}^{G}(a) \cup b
$$

for any $b \in H^{*}(G)$. Of course, this does not determine the cohomology map

$$
H^{*}\left(Q\left(B_{G_{2}(q)}\right)\right) \stackrel{t r^{*}}{\rightarrow} H^{*}\left(Q\left(B_{H}\right)\right)
$$

but it does determine it up to terms involving elements dual to loop sums and terms of the form $Q_{I}(\beta)$.

Note that the image of $H_{*}\left(Q\left(B_{G_{2}(q)}\right)\right.$ in $\mathcal{H}_{4} / \mathcal{H}_{6}$ in $H_{*}\left(Q\left(S^{0}\right)\right)$ is detected by the elements $\sigma_{2}^{i} S q^{1}\left(\sigma_{2}\right)^{j}$. Thus, we need to study the composite map on the $\sigma_{2}^{i}\left(S q^{1}\left(\sigma_{2}\right)^{j}\right.$. The transfer does not preserve cup products but it is natural with respect to the action of the Steenrod algebra, $\mathcal{A}(2)$, and, in low dimensions, the module over $\mathcal{A}(2)$ spanned by these classes is generated by $\sigma_{2}, \sigma_{2}^{3}, \sigma_{2}^{7}$. For our purposes it suffices to determine the transfer just on the images of these elements in $H^{*}\left(M_{12}\right)$. But $\sigma_{2} \mapsto \alpha \in H^{*}\left(M_{12}\right)$ as we have seen. We have $m^{3} n$.

LEMMA 5.11. $\operatorname{tr} \circ \operatorname{res}^{*}(\alpha)=\operatorname{tr} \circ \operatorname{res}^{*}\left(\alpha^{3}\right)=0$ in $H^{*}\left(G_{2}(q)\right)$, while $\operatorname{tr} \circ \operatorname{res}^{*}\left(\alpha^{7}\right)=$

Proof. We have already verified the map on $\alpha^{7}$. It remains to check the others. Since $H^{2}\left(G_{2}(q)\right)=0$ the result follows for $\alpha$. It remains to check $\alpha^{3}$. Since $d_{2}^{3} \epsilon$ $H^{*}\left(V_{5}\right)$ is invariant under $\mathcal{S}_{3}$ it follows that the restriction of $\operatorname{tr}^{*}\left(\alpha^{3}\right)$ to $H^{*}\left(V_{I I}\right)$ is zero. Also, $\sum v \in H^{1}\left(2^{3}\right) v^{3}=0$ so the restriction to $H^{*}\left(V_{I}\right)$ is also zero and the result follows.

COROLLARY 5.12: The image of $\left[S^{14}\right]$ in diagram $(d)$ represents either the class $\sigma^{2}$ or $\sigma^{2}+\bar{\kappa}$ in $\pi_{14}^{s}\left(S^{0}\right)$.

Proof. The projection of the image of $H_{*}\left(Q\left(B_{G_{2}(q)}\right)\right)$ to $\mathcal{H}_{4} / \mathcal{H}_{6}$ via the composition in (5.1) or (5.2) is zero in dimensions $<14$ abd 5.11 shows that it takes the class $\left[G_{2}\right]$ to $\left\{Q_{7}(J) * Q_{7}(J) * 4(-J)\right\}$.

As we have already seen, the Hurewicz image of the sphere in (5.2) has the form $\left[G_{2}\right]+D$ where $D$ is a sum of loop sum decomposables. Moreover, by naturality with 
respect to loop sum, the image of $D$ is contained in $\mathcal{H}_{6}$. Consequently, the spherical class in $\pi_{14}\left(Q\left(S^{0}\right)\right.$ obtained by the composition (5.2) has non-trivial Hurewicz image in $H_{14}\left(Q\left(S^{0}\right)\right)$ of the form $Q_{7}(J) * Q_{7}(J) * 4(-J)$ summed with terms of higher filtration. Consequently, it can only be $\sigma_{7}^{2}$ or $\bar{\kappa}+\sigma_{7}^{2}$, since $\sigma_{14}^{s}\left(S^{0}\right)=2^{2}$ and the Hurewicz image of $\bar{\kappa}$ is trivial in $\mathcal{H}_{4} / \mathcal{H}_{6}$.

6. The proof of Theorem 1.8. We begin by determining the structures of the various images under projection

$$
H^{*}\left(M_{12}\right) \stackrel{\pi_{i}^{*}}{\longrightarrow} H^{*}\left(2^{3}\right)
$$

as $2^{3}$ runs over $V_{1}, V_{3}, V_{5}$ and $i=1,3,5$ respectively.

LEMMA 6.1. The image of $\pi_{5}^{*}$ in $H^{*}\left(V_{5}\right)$ is

$$
\mathbb{F}_{2}\left[d_{2}, d_{3}, d_{4}\right]=\mathbb{F}_{2}\left[d_{4}, d_{6}, d_{7}\right]\left(1, d_{2}, d_{3}, d_{2}^{2}, d_{2} d_{3}, d_{3}^{2}, d_{2}^{4}\right)(1, c)
$$

with relations

$$
\begin{aligned}
c^{2} & =d_{3} c+\dot{d}_{2}^{3}+d_{3}^{2} \\
d_{2}^{2} d_{3} & =d_{7}+d_{3} d_{4}, \\
d_{2}^{3}+d_{3}^{2} & =d_{2} d_{4}+d_{6},
\end{aligned}
$$

In particular it is Cohen-Macaluay over $\mathbb{F}_{2}\left[d_{4}, d_{6}, d_{7}\right]$ on the stated generators.

Proof. First we note that $S q^{1}(c)=\tau^{4}+\tau^{2} \lambda^{2}+\lambda^{4}=d_{2}^{2}, S q^{2}(c)=\tau^{5}+\tau^{4} \lambda+\lambda^{5}$ which in turn is seen to be $d_{2}\left(c+d_{3}\right)$. Thus we have

$$
c^{2}=S q^{3}(c)=S q^{1} S q^{2}(c)=S q^{1}\left(d_{2} c+d_{2} d_{3}\right)=d_{3} c+d_{2}^{3}+d_{3}^{2}
$$

as asserted. This proves the first relation.

The expressions for the $d_{i} i=4,6,7$, given in (1.3) give the relations $d_{2} d_{4}+d_{6}=$ $d_{2}^{3}+d_{3}^{2}, d_{3} d_{4}+d_{7}=d_{2}^{2} d_{3}$ which are sufficient to show that the algebra spanned by $d_{2}, d_{3}, d_{4}, d_{6}, d_{7}$ is just the polynomial algebra $\mathbb{F}_{2}\left[d_{2}, d_{3}, d_{4}\right]$. Consequently the Poincaré series of this algebra is

$$
\begin{aligned}
\frac{1}{\left(1-x^{4}\right)\left(1-x^{3}\right)\left(1-x^{2}\right)} & =\frac{\left(1-x+x^{2}\right)\left(1+x+x^{2}+x^{3}+x^{4}+x^{5}+x^{6}\right)}{\left(1-x^{4}\right)\left(1-x^{6}\right)\left(1-x^{7}\right)} \\
& =\frac{1+x^{2}+x^{3}+x^{4}+x^{5}+x^{6}+x^{8}}{\left(1-x^{4}\right)\left(1-x^{6}\right)\left(1-x^{7}\right)} .
\end{aligned}
$$

Next, if we factor out by the ideal $\left(d_{4}, d_{6}, d_{7}\right)$ this polynomial ring becomes the algebra

$$
\mathbb{F}_{2}\left[d_{2}, d_{3}\right] /\left(d_{2}^{3}+d_{3}^{2}, d_{2}^{2} d_{3}\right)
$$

which has generators (at most) $1, d_{2}, d_{3}, d_{2}^{2}, d_{2} d_{3}, d_{3}^{2}, d_{2}^{4}$. Hence, checking the Poincaré series the result follows.

COROLlaRY 6.2. The image of $\pi_{1}^{*}$ in $H^{*}\left(V_{1}\right)$ is generated by $d_{4}, d_{6}, d_{7}, d_{3}, d_{2} d_{3}$ and as a module over $\mathbb{F}_{2}\left[d_{4}, d_{6}, d_{7}\right]$ is free on the generators

$$
1, d_{3}, d_{2} d_{3}, d_{3}^{2}, d_{2} d_{3}^{2}, d_{3}^{3}, d_{2} d_{3}^{3}
$$

Proof. We embed this algebra into the algebra above. Note that $d_{3}, d_{2} d_{3}, d_{3}^{2}$, are already generators and

$$
d_{2} d_{3}^{2} \equiv d_{2}^{4} \bmod \left(d_{4}, d_{6}, d_{7}\right)
$$


On the other hand we have $d_{3}^{3}=d_{3} d_{6}+d_{2} d_{7}, d_{2} d_{3}^{3}=d_{2} d_{3} d_{6}+d_{2}^{2} d_{7}$ which are independent of the previous generators over $\mathbb{F}_{2}\left[d_{4}, d_{6}, d_{7}\right]$. Finally,

$$
\begin{aligned}
d_{2} d_{3}^{4} & =d_{2} d_{3}^{2} d_{6}+\left(d_{2}^{2} d_{3}\right) d_{7} \\
& =\left(d_{2} d_{3}\right) d_{3} d_{6}+d_{3} d_{4} d_{7}+d_{7}^{2}
\end{aligned}
$$

which shows that this element is already present in the image, so the process stops and we are done.

Finally, we look at the structure of the image of $\pi_{3}^{*}$ in $H^{*}\left(V_{3}\right)$.

LEMma 6.3. The subalgebra of $H^{*}\left(V_{3}\right)$ generated by $d_{4}, d_{6}, d_{7}, h$ has the form $\mathbb{F}_{2}\left[h, d_{4}, d_{6}\right]$ and is Cohen-Macaulay over $\mathbb{F}_{2}\left[d_{4}, d_{6}, d_{7}\right]$. It can be written

$$
\mathbb{F}_{2}\left[d_{4}, d_{6}, d_{7}\right]\left(1, h, h^{2}, h^{3}, h^{4}, h^{5}, h^{6}\right)
$$

with relation $d_{7}=h^{7}+h^{3} d_{4}+h d_{6}$.

Proof. We first verify the relation:

$$
\begin{aligned}
h^{3} d_{4} & =h^{7}+h^{5} d_{2}+h^{4} d_{3}+h^{3} d_{2}^{2} \\
h d_{6} & =h^{5} d_{2}+h^{3} d_{2}^{2}+h^{2} d_{2} d_{3}+h d_{3}^{2}
\end{aligned}
$$

so summing with $h^{7}$ directly gives $d_{7}$. This relation shows that the algebra spanned by $d_{4}, d_{6}, d_{7}, h$ is already spanned by $d_{4}, d_{6}$, and $h$. But it is easy to check that these three elements are transcendentally independent.

Hence, the algebra we are considering is, indeed, $\mathbb{F}_{2}\left[h, d_{4}, d_{6}\right]$ with Poincaré series

$$
\begin{aligned}
P & =\frac{1}{(1-x)\left(1-x^{4}\right)\left(1-x^{6}\right)} \\
& =\frac{1+x+x^{2}+x^{3}+x^{4}+x^{5}+x^{6}}{\left(1-x^{4}\right)\left(1-x^{6}\right)\left(1-x^{7}\right)}
\end{aligned}
$$

On the other hand, from our relation, the algebra is surely spanned as a module over $\mathbb{F}_{2}\left[d_{4}, d_{6}, d_{7}\right]$ by the elements $1, h, h^{2}, h^{3}, h^{4}, h^{5}$ and $h^{6}$, so the Poincaré series above shows that it must be free on these generators and we are done.

Lemma 6.4. Let $P_{i}, i=1,3,5$ be the kernel of projection $H^{*}\left(M_{12}\right) \rightarrow H^{*}\left(V_{i}\right)$, then we have

(1) A generating set for $P_{5}$ over $H^{*}\left(M_{12}\right)$ is

$$
\begin{array}{cccc}
\left(\begin{array}{ccc}
d_{6} & d_{6}+h^{2} d_{4}+h^{6} & 0
\end{array}\right) \\
\left(\begin{array}{ccc}
d_{7} & d_{7} & 0
\end{array}\right) \\
\left(\begin{array}{ccc}
d_{3} & 0 & 0
\end{array}\right)
\end{array}
$$

(2) The image of $P_{5}$ under $\pi_{1}: H^{*}\left(M_{12}\right) \rightarrow H^{*}\left(V_{1}\right)$ is

$$
\left(d_{6}, d_{7}\right) \oplus \mathbb{F}_{2}\left[d_{4}, d_{6}, d_{7}\right]\left(d_{3}, d_{2} d_{3}, d_{3}^{2}, d_{2} d_{3}^{2}, d_{3}^{3}, d_{2} d_{3}^{3}\right)
$$

where $\left(d_{6}, d_{7}\right)$ is the ideal in $\mathbb{F}_{2}\left[d_{4}, d_{6}, d_{7}\right]$ generated by $d_{6}$ and $d_{7}$.

Proof. We check (1) first. The defining relations in the image of $\pi_{5}$ have been seen to be $d_{7}+d_{4} d_{3}+d_{2}^{2} d_{3}=0$, and $d_{6}+d_{4} d_{2}+d_{3}^{2}+d_{2}^{3}=0$. Thus, the elements 
$d_{7}+d_{4} d_{3}+d_{2}^{2} d_{3}, d_{6}+d_{4} d_{2}+d_{3}^{2}+d_{2}^{3}$ together with the remaining generators of $H^{*}\left(M_{12}\right)$ which map to zero in $H^{*}\left(V_{5}\right)$ generate $P_{5}$. This gives the list in (1).

Next we check (2). But this is entirely direct from (1).

Now we study $P_{5}$ in more detail. The generators given above are generators over $H^{*}\left(M_{12}\right)$ so the generators as a module over $\mathbb{F}_{2}\left[d_{4}, d_{6}, d_{7}\right]$ are given by the elements

\begin{tabular}{|c|c|c|}
\hline$d_{6}$ & $d_{6}+h^{2} d_{4}+h^{6}$ & c \\
\hline$d_{7}$ & $d_{7}$ & c \\
\hline$d_{3}$ & 0 & c \\
\hline$d_{2} d_{3}$ & 0 & c \\
\hline$d_{3}^{2}$ & 0 & 0 \\
\hline$d_{2} d_{3}^{2}$ & 0 & c \\
\hline$d_{3}^{3}$ & 0 & 0 \\
\hline$d_{2} d_{3}^{3}$ & 0 & 0 \\
\hline
\end{tabular}

together with products of these elements with powers of the remaining generators,

$$
\left(0, h^{2}, d_{2}\right), \quad\left(0,0, d_{3}\right) \quad\left(0, h^{3}, c\right)
$$

where we can clearly ignore the middle generator. From the relation in $\mathbb{F}_{2}\left[h, d_{4}, d_{6}\right]$ we see that

$$
\begin{aligned}
\left(0, h^{2}, d_{2}\right)\left(d_{6}, d_{6}+h^{2} d_{4}+h^{6}, 0\right) & =\left(0, h^{8}+h^{2} d_{6}+h^{4} d_{4}, 0\right) \\
& =\left(0, h d_{7}, 0\right)
\end{aligned}
$$

so the remaining generators (over $\mathbb{F}_{2}\left[d_{4}, d_{6}, d_{7}\right]$ ) for $P_{5}$ are contained in the following list

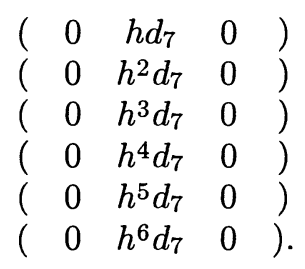

But we easily see that the three generators $\left(d_{6}, h^{6}+h^{2} d_{4}+d_{6}, 0\right),\left(d_{7}, d_{7}, 0\right)$ and

$$
\left(0, h^{6} d_{7}+d_{4} h^{2} d_{7}, 0\right)
$$

together give just two copies of $\mathbb{F}_{2}\left[d_{4}, d_{6}, d_{7}\right]$ since we have an exact sequence

$$
0 \longrightarrow \mathbb{F}_{2}\left[d_{4}, d_{6}, d_{7}\right]\left(d_{7} A+d_{6} B\right) \hookrightarrow \mathbb{F}_{2}\left[d_{4}, d_{6}, d_{7}\right](A, B) \longrightarrow\left(d_{6}, d_{7}\right) \longrightarrow 0
$$

where $A \mapsto d_{6}$ and $B \mapsto d_{7}$ in the ideal $\left(d_{6}, d_{7}\right) \subset \mathbb{F}_{2}\left[d_{4}, d_{6}, d_{7}\right]$. Thus the last generator in the list above may be suppressed Moreover, by the same imbedding technique as was used in the second lemma we see that the remaining needed generators $\left(0, h^{i} d_{7}, 0\right)$, $1 \leq i \leq 6$ are independent and generate a free module over $\mathbb{F}_{2}\left[d_{4}, d_{6}, d_{7}\right]$. However, since the generators $\left(d_{6}, h^{6}+h^{2} d_{4}+d_{6}, 0\right)$ and $\left(d_{7}, d_{7}, 0\right)$ are not needed, we see that $\left(0, h^{7} d_{7}, 0\right) \sim\left(0, d_{7}^{2}, 0\right)$ is also a free generator, and adding this in gives a complete list of generators for $H^{*}\left(M_{12}\right)$ over $\mathbb{F}_{2}\left[d_{4}, d_{6}, d_{7}\right]$.

\section{REFERENCES}

[AM] A. Adem, R.J. Milgram, Cohomology of Finite Groups, Grundlehren der Math. 309, Springer-Verlag (1995). 
[AMM1] A. Adem, J. Maginnis, R.J. Milgram, The geometry and cohomology of the Mathieu group $M_{12}, J$. of Algebra, 139(1991), 90-133.

[AMM2] A. Adem, J. Maginnis, R.J. Milgram, Symmetric invariants and cohomology of groups, Math. Ann., 287(1990), 391-411.

[BW] D. Benson, C. Wilkerson, Finite simple groups and Dickson invariants, Homotopy Theory and its Applications (Cocoyoc 1993), 39-50, Contemp. Math 188, A.M.S., 1995.

[M] R.J. Milgram, On the geometry and cohomology of the simple groups $G_{2}(q)$ and ${ }^{3} D_{4}(q)$, II, Group Representations: Cohomology, Group Actions, and Topology, Proc. Sympos. Pure Math., Vol 63, A.M.S. (1998), pp. 397 - 418.

[MO] R.J. Milgram, C. Overton, The stable splitting of $M_{12}$, Preprint, Stanford University, (1996).

[O] C. Overton, Thesis, Stanford University, 1996.

[R] N. RaY, Invariants of reframed manifolds, Proc. Lond. Math. Soc., (3) 39 (1979), 253-275. 